\title{
Innovations and Challenges by Applying Sublingual Laser Blood Irradiation in Juvenile Idiopathic Arthritis
}

\author{
Laura Marinela Ailioaie, ${ }^{1,2}$ Gerhard Litscher, ${ }^{3}$ Michael Weber, ${ }^{4}$ Constantin Ailioaie, ${ }^{2,5}$ \\ Daniela Litscher, ${ }^{3}$ and Dragos Andrei Chiran ${ }^{2,6}$ \\ ${ }^{1}$ Department of Medical Physics, "Alexandru Ioan Cuza” University, 11 Carol I Boulevard, 700506 Iasi, Romania \\ ${ }^{2}$ Laser Clinic, 83 Arcu Street, 700135 Iasi, Romania \\ ${ }^{3}$ Research Unit for Complementary and Integrative Laser Medicine, Research Unit of Biomedical Engineering in Anesthesia and \\ Intensive Care Medicine and TCM Research Center Graz, Medical University of Graz, Auenbruggerplatz 29, 8036 Graz, Austria \\ ${ }^{4}$ Institute for Laser Therapy and Acupuncture, Sohnreystraße 6, 37697 Lauenförde, Germany \\ ${ }^{5}$ Second Pediatric Clinic, "Saint Mary" Emergency Hospital for Children, 62 Vasile Lupu Street, 700309 Iasi, Romania \\ ${ }^{6}$ Faculty of Medicine, "Grigore T. Popa" University of Medicine and Pharmacy, 16 Universitatii Street, 700115 Iasi, Romania
}

Correspondence should be addressed to Gerhard Litscher; gerhard.litscher@medunigraz.at

Received 12 May 2014; Accepted 30 May 2014; Published 17 June 2014

Academic Editor: Lu Wang

Copyright (c) 2014 Laura Marinela Ailioaie et al. This is an open access article distributed under the Creative Commons Attribution License, which permits unrestricted use, distribution, and reproduction in any medium, provided the original work is properly cited.

\begin{abstract}
Sublingual laser blood irradiation (SLBI) was applied into a randomized, single-blind, placebo-controlled study in juvenile idiopathic arthritis (JIA), aimed at inducing disease remission. 105 children with JIA, without an adequate response to classical treatment, were administrated a disease modifying drug (Methotrexate) and were randomly assigned to three groups. Group I (36 patients) received SLBI with the Weberneedle Lasershower Mouth Applicator with three wavelengths $(635 \mathrm{~nm}, 536 \mathrm{~nm}$, and $405 \mathrm{~nm}$ ), $5 \mathrm{~mW}$ maximum output power each, in continuous mode, simultaneously, for 20 minutes daily, 7 successive sessions per month, repeated every 7 weeks, for three times. Group II (36 patients) received placebo SLBI. Group III (33 patients) received only treatment with Methotrexate. Evaluation was performed using American College of Rheumatology Pediatric criteria (ACR Pedi) at study enrollment and at $8,16,24$, and 48 weeks. At the end of study, there was an improvement of the ACR Pedi 30 by $86.11 \%$ in SLBI group compared to only $61.11 \%$ in Group II, respectively, and $60.6 \%$ in Group III $(P=0.001)$, with significant statistical differences. SLBI has reduced the pain, lowered the number of articulations with movement limitation, increased the quality of life, and made it possible to avoid the administration of biological agents.
\end{abstract}

\section{Introduction}

A generation ago, children with arthritis faced a lifetime of pain and disability. Juvenile idiopathic arthritis (JIA), an umbrella term covering multiple distinct categories, previously called juvenile rheumatoid arthritis until recent reclassification, is one of the most common chronic diseases of childhood, featuring arthritis of unknown etiology $[1,2]$.

JIA is a heterogeneous autoimmune chronic inflammatory disease which occurs before the age of 16 , characterized by chronic pain with persistent arthritis, for at least six weeks, in one or more joints, and/or extra-articular manifestations, with a wide spectrum of presentations and subsets, and can lead to severe disability, due to periods of remission and flares [3].

This heterogeneity with respect to clinical, demographic, and genetic features among the JIA subtypes is translating into heterogeneity in the responses to treatment [2].

Arthritis with synovial proliferation, triggered by the secretion of proinflammatory factors and the formation of granular tissue with monocytes, macrophages, lymphocytes, and other immune cells, will lead to progressive destruction of the articular structures [4].

More than one-third of children have ongoing active disease into adulthood with sequelae from chronic inflammation [5]. 
Patients with moderate or severe forms of JIA tend to have a worse prognosis, even with the early use of disease modifying antirheumatic drugs (DMARDs). These patients have considerable morbidity from joint damage, osteoporosis, growth retardation, psychosocial morbidity, reduced quality of life, and educational or employment disadvantage [6].

Chronic pain has a large and wholly negative impact on the physical and psychological well-being of patients and their family. Childhood chronic pain is a modern public health disaster, which is only now coming to light [4].

Today, there are a multitude of treatment options, including biological agents and complementary alternatives $[1,7]$. Goals of any JIA management are to control inflammation, relieve pain, prevent or control joint damage, and maximize joint and body function.

In the last years, an early aggressive therapy with a combination of Methotrexate and biological agents to sustain clinically inactive disease in children with JIA, more often than treatment with Methotrexate alone, was applied [8].

Unfortunately, side effects of biological agents, which suppress the immune system (and thus inflammation related to arthritis), can have an increased risk of infection [9] and others.

So, the newest treatment goals in JIA should aim to induce remission with the least toxicity from medications with hopes of a prolonged remission, particularly important in pediatrics, in which the rate of progression and the onset of debility can be rapid, affecting the quality of life of the future adult.

New clinical innovations are challenging advances in medical treatments using intravenous laser blood irradiation (ILBI) [10].

Recently, ILBI and the biological agent etanercept were applied in a medical effort to achieve early control of inflammation, to prevent joint damage or loss of function, and to decrease pain and systemic damage in JIA [11].

Also, a synergistic anti-inflammatory effect of ILBI was proved, if applied additionally in combination with tocilizumab, in case of a therapy-resistant severe form of JIA [12].

Juvenile arthritis imposes a societal burden of significant health care costs [13], and the expensive biological agents are increasingly prescribed, earlier in the disease and in patients with JIA with lower disease activity. These changes are accompanied by only short-term better disease outcomes [14].

New methods to modulate the activity of the immune system in JIA and to enhance the efficacy of medication are extremely necessary. These should bring unique and truly remarkable pain relief; they should be noninvasive, nonpharmaceutical, not addictive, with no significant side effects, or even free of side effects, using no injections, of which the children are afraid.

Aim of this study was to introduce a new technique in the field of photoenergy and to investigate the effects of complex photobiomodulation, that is, sublingual laser blood irradiation (SLBI) (transmucosal irradiation under the tongue) in JIA patients, with different wavelengths that scan the whole visible spectrum.
Blood circulation is very abundant on children's dorsal tongue, so laser irradiation of this area can bring great benefits, being noninvasive and painless, compared with the intravenous laser blood irradiation (ILBI) in this pathology.

\section{Patients and Methods}

2.1. Study Design. Between 01.02.2012 and 01.02.2014, 105 children were included in a prospective, randomized, singleblind, placebo-controlled study at the Second Pediatric Clinic of the St. Mary Emergency Hospital for Children, Iasi, Romania. Eligible patients were 7 to 16 years of age (mean age \pm SD: $10.2 \pm 4.3$ years), diagnosed with different subtypes of active juvenile idiopathic arthritis (Table 1), taking into account the classification criteria for JIA established by the International League of Associations for Rheumatology (ILAR) [15].

Patients had persistent disease ( $\geq 6$ months) and inadequate responses to nonsteroidal anti-inflammatory drugs (NSAIDs) and glucocorticoids. All the patients were administrated a disease modifying drug, Methotrexate, in a dose of $0.6 \mathrm{mg} / \mathrm{kg}$, one intramuscular or subcutaneous administration per week (maximum $20 \mathrm{mg} /$ week), and were randomly assigned to three groups for the sublingual irradiation study, as a complementary method of therapy. All patients receiving Methotrexate were also prescribed $1 \mathrm{mg} /$ day folic acid.

To eliminate bias in the treatment assignment, participants were randomly allocated to one of three study groups with equal probability using a randomized design. Group I, of 36 patients, received sublingual laser blood irradiation with the Weberneedle Lasershower Mouth Applicator, from the Weber Medical laser device with three wave lengths $(635 \mathrm{~nm}, 536 \mathrm{~nm}$, and $405 \mathrm{~nm}), 5 \mathrm{~mW}$ maximum output power each, in continuous mode, simultaneously, 20 minutes each session. The patients underwent one session daily, 7 successive sessions per month, repeated every 7 weeks for three times, for a total of 24 weeks, that is, with a 7-week interval between each set of photobiomodulations. Group II, of 36 patients, received placebo sublingual laser blood irradiation. Group III, 33 patients, received only treatment with Methotrexate.

All the patients could benefit from steroid therapy at a maximum dose of $0.5 \mathrm{mg} / \mathrm{kg} / \mathrm{day}$, analgesics, and individualized physical therapy.

All patients and their parents or legal guardian were fully informed of all aspects of this health care related research and all their questions were answered. The possible side effects and benefits of photobiomodulation, that is, SLBI, were fully explained to the enrolled patients and their parents. The patients and their parents were fully aware of the existence of the placebo group and they were guaranteed that the ones who would be in the placebo group would be offered, at the end of the study, a real SLBI trial, identical with the one the patients in Group I had, should they wish. A written informed consent was signed by parents or legal guardian of each JIA patient included in the study.

This research was in accordance with the Declaration of Helsinki on ethical principles for medical research involving 
TABLE 1: Baseline demographic and clinical characteristics of the JIA patients at the initiation of the study.

\begin{tabular}{|c|c|c|c|c|}
\hline Characteristics & $\begin{array}{l}\text { Group I } \\
(n=36)\end{array}$ & $\begin{array}{c}\text { Group II } \\
(n=36)\end{array}$ & $\begin{array}{c}\text { Group III } \\
(n=33)\end{array}$ & Statistical significance \\
\hline \multicolumn{5}{|l|}{ Subtype JIA } \\
\hline RF negative polyarthritis-number (\%) & $11(30.55)$ & $11(30.55)$ & $10(30.30)$ & {$[\mathrm{NS}]$} \\
\hline RF positive polyarthritis_-number (\%) & $7(19.44)$ & $9(25.00)$ & $6(18.18)$ & {$[\mathrm{NS}]$} \\
\hline Enthesitis-related arthritis-number (\%) & $9(25.00)$ & $8(22.22)$ & $6(18.18)$ & {$[\mathrm{NS}]$} \\
\hline Oligoextensive-number (\%) & $8(22.22)$ & $7(19.44)$ & $10(30.30)$ & {$[\mathrm{NS}]$} \\
\hline Psoriatic arthritis-number (\%) & $1(2.77)$ & $1(2.77)$ & $1(3.03)$ & {$[\mathrm{NS}]$} \\
\hline Female sex-number (\%) & $19(52.77)$ & $17(47.22)$ & $18(54.54)$ & {$[\mathrm{NS}]$} \\
\hline Age-yrs & $10.0 \pm 4.3$ & $10.6 \pm 4.4$ & $10.0 \pm 4.1$ & {$[\mathrm{NS}]$} \\
\hline Weight-kg & $32.7 \pm 15.6$ & $31.8 \pm 15.8$ & $31.9 \pm 16.1$ & {$[\mathrm{NS}]$} \\
\hline Duration of disease $-\mathrm{yrs}$ & $3.1 \pm 2.0$ & $3.2 \pm 2.3$ & $3.2 \pm 1.0$ & {$[\mathrm{NS}]$} \\
\hline \multicolumn{5}{|l|}{ Prior use of DMARDs (mean dose) } \\
\hline Methotrexate (mg/wk) & $18.4 \pm 2.1$ & $18.2 \pm 2.1$ & $18.9 \pm 2.0$ & {$[\mathrm{NS}]$} \\
\hline Sulfasalazine (g/day) & $1.0 \pm 0.5$ & $1.2 \pm 0.50$ & $1.0 \pm 0.25$ & {$[\mathrm{NS}]$} \\
\hline Hydroxychloroquine (mg/day) & $200 \pm 0.5$ & $190 \pm 0.5$ & $200 \pm 0.5$ & {$[\mathrm{NS}]$} \\
\hline Oral glucocorticoid $(\mathrm{mg} / \mathrm{kg} / \mathrm{day})^{*}$ & $0.49 \pm 0.18$ & $0.49 \pm 0.19$ & $0.49 \pm 0.17$ & {$[\mathrm{NS}]$} \\
\hline
\end{tabular}

NS: nonsignificant difference between the groups, $P$ value $\geq 0.05$. SD: standard deviation. RF: rheumatoid factor. ${ }^{*}$ For each oral glucocorticoid used, the prednisone equivalent was calculated.

human subjects. Ethical approval of the medical institution mentioned above was given.

The most important inclusion criterion in the study was patients with persistent active JIA (with course $\geq 6$ months prior to study entry) and inadequate responses to nonsteroidal anti-inflammatory drugs (NSAIDs) and glucocorticoids, assigned to one of the following JIA subtypes: rheumatoid factor positive or negative JIA, enthesitis related arthritis, extended oligoarticular JIA, and polyarticular juvenile psoriatic arthritis. Other inclusion criteria were being aged between 7 and 16 years at the study enrollment and no evidence of current, active tuberculosis (TB) or old, inactive TB.

Exclusion criteria were patients having active infections, risk of sepsis, a history of or ongoing chronic or recurrent infectious disease (hepatitis, with B or C virus, HIV, etc.), a known history of demyelinating diseases such as multiple sclerosis, a history of malignancies or lymphoproliferative disease, including lymphoma, and active uveitis and pregnant or sexually active female patients without using effective contraception. Other exclusion criteria were related to SLBI, such as history of epilepsy or sensitivity to light.

The study was conducted over a 48 -week period and had two parts: the SLBI phase (24 weeks) and a 24-week extension phase to follow the evolution of the patients; that is, it was a 12-month follow-up study.

The administration should be discontinued in case of adverse effects, such as dysphagia, nausea, vomiting, mucous ulcerations, headache, rash, and abdominal pain.

2.2. Sublingual Laser Blood Irradiation. Laser equipment consisted of Weberneedle Endolaser with the Weberneedle Lasershower Mouth Applicator (Figures 1 and 2).
All the patients in Group I received SLBI with three wavelengths ( $635 \mathrm{~nm}, 536 \mathrm{~nm}$, and $405 \mathrm{~nm}$ ), $5 \mathrm{~mW}$ maximum output power each, in continuous mode, simultaneously, for 20 minutes daily, 7 successive sessions per month, repeated every 7 weeks, for three times.

For hygienic reasons, a transparent foil is wrapped tightly around the tip of the probe (Figure 3). Its exact placement underneath the tongue is important, as the blood vessels are located there.

The SLBI protocol was the same for both Groups I and II, but the placebo patients did not effectively receive the laser radiation through the Weberneedle Lasershower Mouth Applicator. None of the patients knew if they actually received laser or placebo, as they were asked to relax with eyes closed and wear special goggles (Figure 2).

2.3. Assessment and Outcomes. The patients were evaluated using the ACR (American College of Rheumatology) Pediatric core set for improvement in JIA, which includes the following six variables: physician's global assessment of disease activity (with scores ranging from 0 to 100 indicating the level of active disease), the parent's or patient's global assessment of overall well-being (with scores ranging from 0 to 100 indicating the level of active disease), the physical function (as assessed with the use of the Disability Index of the Childhood Health Assessment Questionnaire (CHAQDI), with scores ranging from 0 to 3 indicating the level of disability), the number of joints with active arthritis, the number of joints with limited range of motion, and the erythrocyte sedimentation rate (ESR normal values $<15 \mathrm{~mm} / \mathrm{h}$ ) [15-19].

The ACR Pediatric 30 (ACR Pedi 30) response represents a minimum of $30 \%$ improvement from baseline in a minimum of three out of six components, with a worsening by 


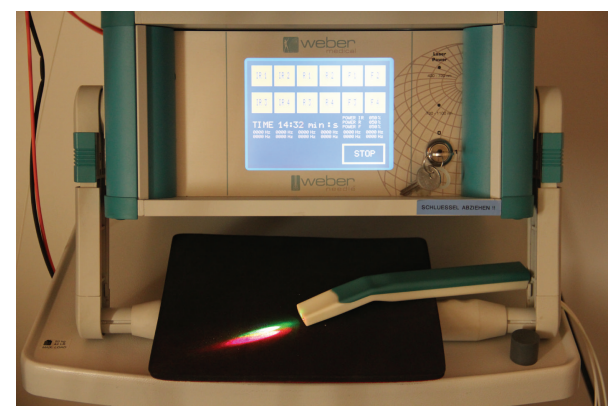

FIGURE 1: Weberneedle Endolaser (identical systems are available at the Medical University of Graz and the St. Mary Emergency Hospital for Children, Iasi). The following wavelengths were used for sublingual laser treatment: $630 \mathrm{~nm}$ (red), $536 \mathrm{~nm}$ (green), and $405 \mathrm{~nm}$ (violet).

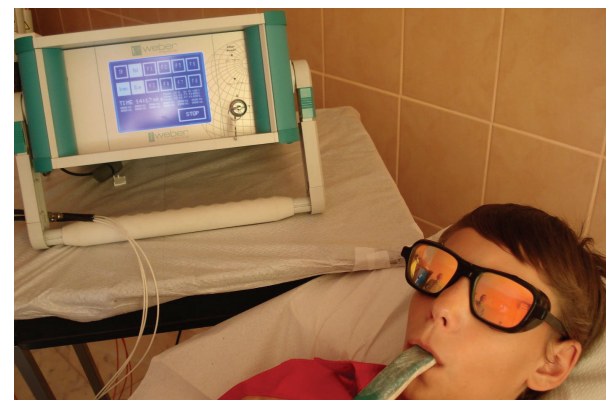

Figure 2: Patient during treatment with the Weberneedle Lasershower Mouth Applicator, from Weber Medical GmbH, Lauenförde, Germany.

$>30 \%$ in no more than one component. The ACR Pediatric 50 (ACR Pedi 50) response requires 50\% improvement in three out of six components with worsening of $30 \%$ in no more than one component. The ACR Pediatric 70 (ACR Pedi 70) requires $70 \%$ improvement in three out of six components with worsening of $30 \%$ in no more than one component. The ACR Pediatric 90 (ACR Pedi 90) is also defined as $90 \%$ improvement in a minimum of three core set variables with worsening of one variable by no more than $30 \%$ [16].

Clinical assessment and the laboratory findings were performed at study entry (baseline characteristics) (Table 1) and at every 8-week interval of time, before starting each new SLBI set of sessions, for three times, and at 48 weeks (end of study).

The qualified staff who carried out the SLBI knew about the patient being assigned to one group or another, but all the necessary measures were taken so as not to influence the patients' outcome.

In order to avoid any bias of the results, as a singleblind study, the certified physical therapists who tested the functional assessment of the joints, included in the ACR Pedi core set data, did not know to which particular study group the patients belonged.

Appropriate statistical analyses were employed. The data in this study were analyzed at the initiation of the therapy, at 8 weeks, 16 weeks, and 24 weeks, and after 12 months of

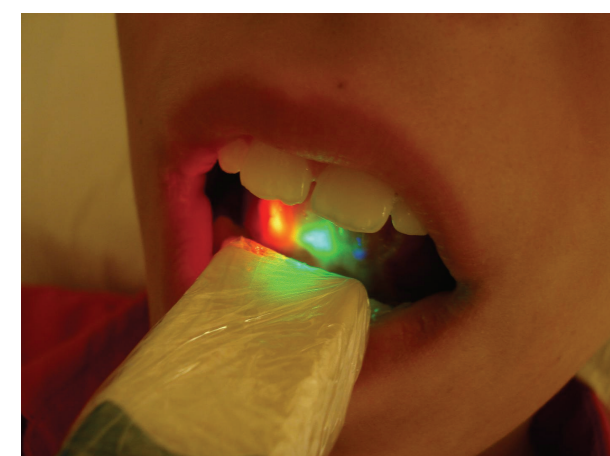

FIGURE 3: Sublingual laser blood irradiation technique.

treatment. The statistical analysis followed the comparison of the groups, performing $t$-student test. The median values and the standard deviation were calculated for all quantifiable data.

A value of $P<0.05$ was considered statistically significant, and a value of $P \geq 0.05$ was considered nonsignificant. The basic statistical analysis and chart generation were done using MS Excel 2010 software.

\section{Results}

All 105 participants enrolled in the study completed the study.

Baseline demographic and clinical variables at entry are shown in Table 1. No significant entry differences existed between the three groups.

At the first evaluation, after 8 weeks from the initiation of this trial, the patients treated with SLBI already displayed a better improvement in all six parameters of the ACR core set data compared with the placebo group, or Group III, respectively.

At 8-week assessment phase, with respect to the ACR Pedi criteria, $61.11 \%$ of the patients in Group I, compared to only $33.33 \%$ of the patients in Group II and $30.30 \%$ of the patients in Group III, respectively, fulfilled the Pedi 30 response (Figures 4, 5, and 6). No patient met the ACR Pedi 90 response at this phase.

Improvement in Group I was even better at 16-week assessment, where JIA ACR30/50/70/90 responses achieved were $72.22 \%, 41.66 \%, 19.44 \%$, and $5.55 \%$, respectively, compared with only $33.33 \%, 13.88 \%, 2.77 \%$, and $0.00 \%$ JIA ACR30/50/70/90 responses in the placebo group, or $36.36 \%$, $18.18 \%, 3.03 \%$, and $0.00 \%$ JIA ACR30/50/70/90 responses in Group III, respectively (Figures 4-6).

Patients given SLBI had a greater chance of achieving JIA ACR30/50/70/90 responses at week 24 in comparison with the placebo patients or the Methotrexate group (Table 2).

The differences in the proportions of SLBI patients and placebo patients or Methotrexate group at each JIA ACR response level were statistically significant $(P<0.001)$.

The whole evolution of the ACR core outcome variables reflected in the JIA ACR30/50/70/90 responses in each group at 8 weeks, 16 weeks, 24 weeks, and 48 weeks, respectively, is represented in Figures 4-6. 


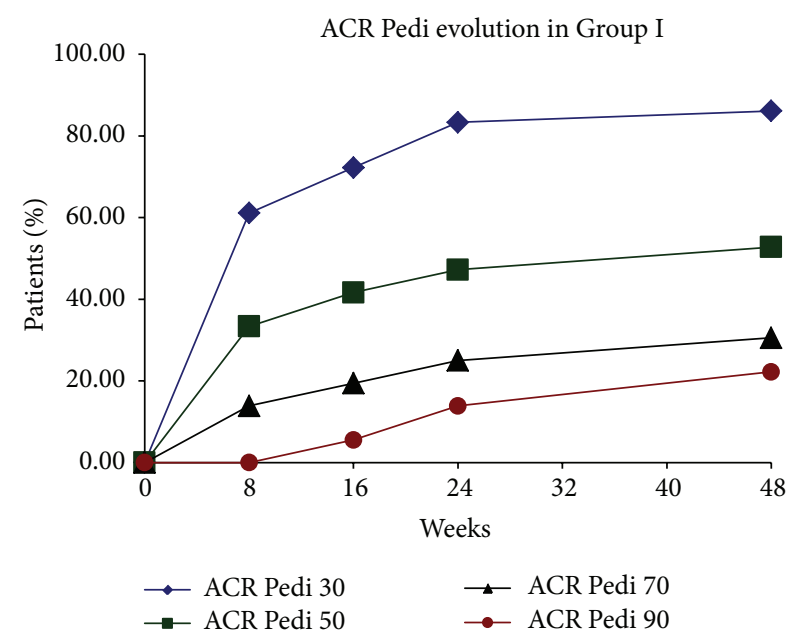

FIgUre 4: Evolution of ACR Pedi 30/50/70/90 responses in SLBI group.

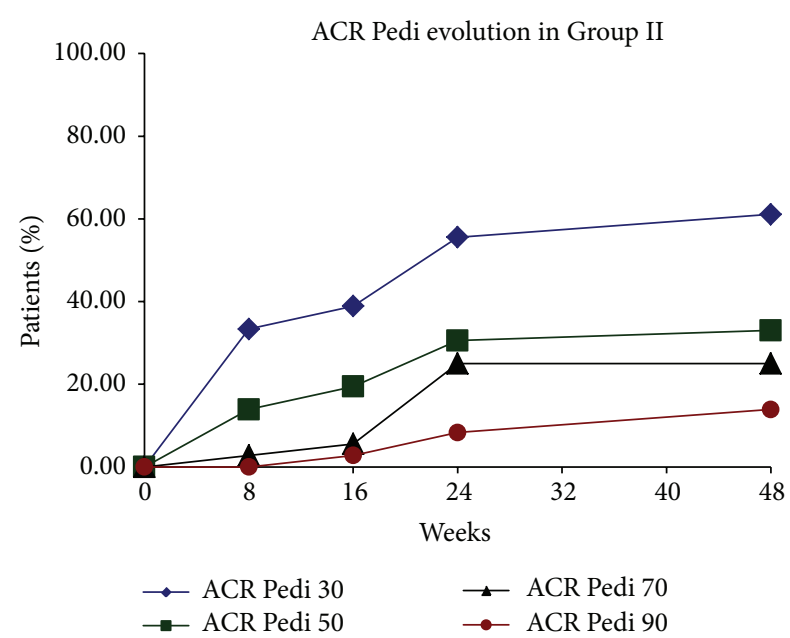

Figure 5: Evolution of ACR Pedi 30/50/70/90 responses in placebo group.

At the end of the study, the proportion of responders showing the best ACR Pedi responses was higher in patients receiving SLBI, compared with those in Groups II and III.

The efficacy of SLBI proved to trigger a positive evolution of the individual ACR core outcome variables, as presented in Table 2.

At the final evaluation, the SLBI patients continued to display a more significant improvement in comparison with the patients in the other two groups, in all aspects encompassed in the ACR core set data.

All the patients completed the study and were closely monitored for possible side effects of SLBI and/or drugs. There were no side effects of laser therapy and no patient manifested any major adverse reactions to medication.

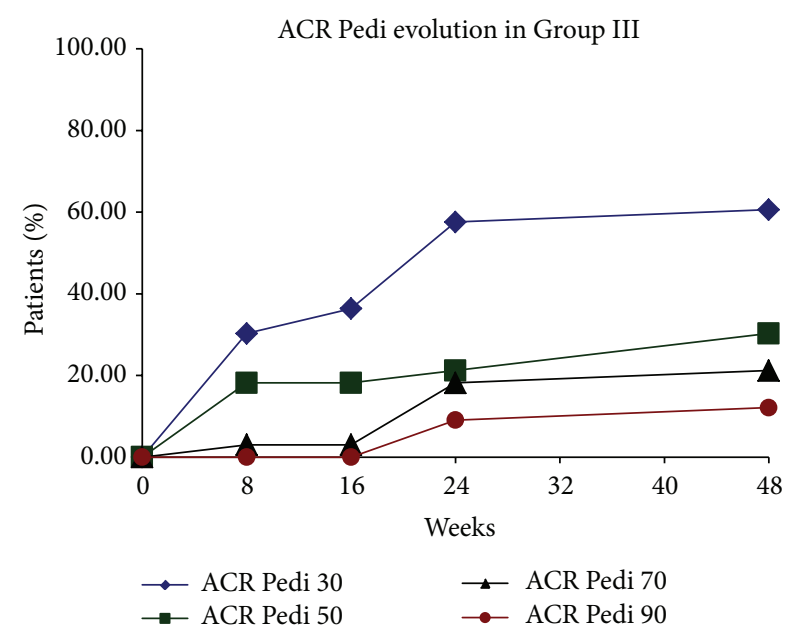

FIgURE 6: Evolution of ACR Pedi 30/50/70/90 responses in Group III.

\section{Discussions}

Children with chronic diseases (such as juvenile arthritis) often have difficulty following a treatment program consistently. No one likes to take medications, do special exercises, or wear splints over a long period of time.

Nowadays, the treatment of juvenile idiopathic arthritis became a revolution in care, because there are a multitude of treatment options, including a variety of biologics targeting key cytokines and other inflammatory mediators. Among the conventional disease modifying antirheumatic drugs, Methotrexate remains first-line therapy for most children with juvenile idiopathic arthritis due to its long track record of safety and effectiveness in the management of peripheral arthritis [1].

Methotrexate (MTX) is an antimetabolite and antifolate drug, used in treatment of cancer and some immunemediated inflammatory diseases [20].

Although MTX was originally designed as a chemotherapy drug (in high doses), in low doses it is a generally safe and well-tolerated drug in the treatment of JIA.

Folic acid is needed for the de novo synthesis of the nucleoside thymidine, required for DNA synthesis. Also, folate is needed for purine base synthesis, so all purine syntheses will be inhibited. MTX, therefore, inhibits the synthesis of DNA, RNA, thymidylates, and proteins [21].

For the treatment of arthritis, multiple mechanisms appear to be involved including the inhibition of enzymes involved in purine metabolism, leading to an accumulation of adenosine; inhibition of $\mathrm{T}$ cell activation and suppression of intercellular adhesion molecule expression by $\mathrm{T}$ cells; increasing CD95 sensitivity of activated T cells; inhibition of methyltransferase activity, leading to (de)activation of enzyme activity relevant to immune system function [22].

Photobiomodulation or previously called photobiostimulation has been clinically investigated for more than 30 years in different pathologies. It was proved as noninvasive, as a valuable alternative, or adjunct, to drugs. Old protocols and 
TABLE 2: Evolution from baseline of ACR core set data at the end of the sublingual laser blood irradiation.

\begin{tabular}{|c|c|c|c|c|c|c|}
\hline \multirow{2}{*}{ Variable } & \multicolumn{2}{|c|}{ Group I $(n=36)$} & \multicolumn{2}{|c|}{ Group II $(n=36)$} & \multicolumn{2}{|c|}{ Group III $(n=33)$} \\
\hline & Baseline & Weeks 24 & Baseline & Weeks 24 & Baseline & Weeks 24 \\
\hline ACR Pedi 30 response number (\%) & - & $30(83.33)$ & - & $20(55.55)$ & - & $19(57.57)$ \\
\hline \multicolumn{7}{|c|}{ ACR core set of variables } \\
\hline $\begin{array}{l}\text { Number of joints with active } \\
\text { arthritis }\end{array}$ & $7.58 \pm 2.32$ & $\begin{array}{l}3.94 \pm 1.14 \\
(P=0.001)\end{array}$ & $7.5 \pm 1.16$ & $\begin{array}{l}6.83 \pm 1.23 \\
(P=0.02)\end{array}$ & $7.42 \pm 2.66$ & $\begin{array}{l}6.66 \pm 1.21 \\
(P=0.13)\end{array}$ \\
\hline $\begin{array}{l}\text { Number of joints with limited range } \\
\text { of motion }\end{array}$ & $17.63 \pm 5.31$ & $\begin{array}{l}9.30 \pm 2.81 \\
(P=0.001)\end{array}$ & $17.69 \pm 14.6$ & $\begin{array}{l}13.80 \pm 8.00 \\
(P=0.16)\end{array}$ & $17.09 \pm 3.66$ & $\begin{array}{c}15.46 \pm 2.65 \\
(P=0.04)\end{array}$ \\
\hline $\begin{array}{l}\text { Physician's global assessment of } \\
\text { overall disease activity }\end{array}$ & $56.11 \pm 10.76$ & $\begin{array}{l}31.25 \pm 9.05 \\
(P=0.001)\end{array}$ & $55.15 \pm 13.22$ & $\begin{array}{c}47.87 \pm 12.69 \\
(P=0.01)\end{array}$ & $56.66 \pm 7.52$ & $\begin{array}{c}51.46 \pm 9.85 \\
(P=0.01)\end{array}$ \\
\hline $\begin{array}{l}\text { Parent of patient global assessment } \\
\text { of overall well-being }\end{array}$ & $58.05 \pm 1.04$ & $\begin{array}{r}33.88 \pm 0.33 \\
(P=0.001)\end{array}$ & $52.22 \pm 13.01$ & $\begin{array}{l}46.66 \pm 13.90 \\
(P=0.09)\end{array}$ & $60.30 \pm 11.69$ & $\begin{array}{c}53.46 \pm 14.9 \\
(P=0.03)\end{array}$ \\
\hline CHAQ-DI score & $1.69 \pm 0.68$ & $\begin{array}{l}1.05 \pm 0.15 \\
(P=0.001)\end{array}$ & $1.55 \pm 0.56$ & $\begin{array}{l}1.22 \pm 0.50 \\
(P=0.01)\end{array}$ & $1.50 \pm 0.54$ & $\begin{array}{l}1.20 \pm 0.60 \\
(P=0.03)\end{array}$ \\
\hline $\operatorname{ESR}(\mathrm{mm} / \mathrm{hr})$ & $42.38 \pm 23.35$ & $\begin{array}{l}17.58 \pm 0.98 \\
(P=0.001)\end{array}$ & $42.22 \pm 6.92$ & $\begin{array}{c}38.55 \pm 6.85 \\
(P=0.02)\end{array}$ & $41.83 \pm 10.28$ & $\begin{array}{c}36.80 \pm 6.50 \\
(P=0.02)\end{array}$ \\
\hline
\end{tabular}

laser devices had marginal success in the past. New lasers and new protocols may have increased success with many types of pain. The pathogenic mechanisms of pain in children with rheumatic diseases are multifactorial and only treatment of the disease itself is not sufficient to alleviate chronic pain [23].

Aims of this study were to provide new methods and protocols to accelerate JIA remission and to be successful at a substantially lower cost, to become accessible to JIA sufferers across the globe, that is, to fulfill criteria of growth and expansion.

Because the pathogenesis of rheumatic diseases in children is multifactorial, the management should be interdisciplinary, consisting of both pharmacologic and nonpharmacologic interventions.

Many other factors besides disease activity contribute to the pain experience in children with arthritis. More complex pain treatments should be considered in those children who continue to have significant residual pain.

There is a lack of generally accepted detailed treatment guidelines for pain management in children with JIA. Given our knowledge of the disease at present, in order to produce successful results regarding chronic pain, it is essential not only to rely on allopath medicine but also to take advantage of the perfect combination between it and the complementary methods, in a holistic approach.

Photobiostimulation is a natural treatment that provides the living cells with an energy source in the form of photons. Many diseases or dysfunctional problems of a bodily system or organ can be successfully treated with this new technology.

As mentioned in the introduction, SLBI (transmucosal irradiation under the tongue) is a noninvasive innovation and has the potential to treat many diseases. However, to the best of our knowledge, this study is the first in children. Application of the different lasers is very easy, and the adverse effects (inflammation) are very few compared to, for example, needle acupuncture on the body or the tongue. In our opinion, there are several indications for SLBI; it especially may be an alternative for invasive laser blood irradiation.
Absorption of the photonic energy from the therapeutic laser light is in the visible red and infrared spectrum. Laser is absorbed by the chromophores in the target tissue. Parameters that determine effective tissue response to laser are the wavelength, depth of penetration (power and time), and number of photons (power and time). Generally speaking, laser light causes a photochemical effect on the target tissue, being absorbed by chromophores on cell membranes and mitochondria, decreasing pain and inflammation, and stimulating healing processes. State-of-the-art laser therapy was radically changed in recent years due to the implementation of new high-tech treatment methods and complex devices (e.g., Weber Medical GmbH, Lauenförde, Germany) in an integrative laser medicine field [23].

Low-level laser (light) therapy (LLLT) is a fast-growing technology used to treat a multitude of conditions that require stimulation of healing, relief of pain and inflammation, and restoration of function. LLLT, phototherapy, or photobiomodulation refers to the use of photons at a nonthermal irradiance to alter biological activity. The mechanism associated with the cellular photobiostimulation by LLLT is not yet fully understood. From observation, it appears that LLLT has a wide range of effects at the molecular, cellular, and tissue levels [24]. The basic biological mechanism behind the effects of LLLT is thought to be through absorption of red and NIR light by mitochondrial chromophores, in particular cytochrome c oxidase (CCO), which is contained in the respiratory chain located within the mitochondria [25-27], and perhaps also by photoacceptors in the plasma membrane of cells. Consequently, a cascade of events occurs in the mitochondria, leading to biostimulation of various processes [24].

Blood circulation is very abundant on children's dorsal tongue, so laser irradiation of this area can bring great benefits, being noninvasive and painless. For the treatment of arthritis, it seems that Methotrexate appears to be involved in multiple mechanisms including the inhibition of enzymes involved in purine metabolism, leading to accumulation 
of adenosine. Other triggered mechanisms by MTX are inhibition of T cell activation and suppression of intercellular adhesion molecule expression by $\mathrm{T}$ cells, increasing CD95 sensitivity of activated T cells, and inhibition of methyltransferase activity, leading to (de)activation of enzyme activity relevant to immune system function [22].

Adenosine plays an important role in biochemical processes, such as energy transfer-as adenosine triphosphate (ATP) and adenosine diphosphate (ADP) - as well as in signal transduction as cyclic adenosine monophosphate (cAMP). Adenosine also plays a role in regulation of blood flow to various organs through vasodilatation. Adenosine is an endogenous purine nucleoside that modulates many physiological processes. Thus, with regard to stress or injury, the function of adenosine is primarily that of cytoprotection preventing tissue damage during instances of hypoxia, ischemia, and so forth. Activation of $\mathrm{A}_{2 \mathrm{~A}}$ receptors produces a constellation of responses that in general can be classified as anti-inflammatory [28].

Anti-inflammatory effect of MTX may be due to its stimulation of adenosine release. Meantime, photobiostimulation may synergistically potentate the cascade of biochemical processes linked to the energy transfer at mitochondrial level (ATP, ADP, and cAMP). One of the most important effects of LLLT is that it accelerates ATP production, by increasing metabolism at the cellular level and enabling the ischemic cells in the damaged tissue to heal and to attain their normal functions.

The results of our study, reflected in the very good ACR Pedi responses in SLBI group, proved that photobiomodulation and MTX are acting synergistically.

Our hypothesis is that if we think of time in nanoseconds, a possible picture of the complex processes triggered inside the cells by both MTX and photobiomodulation in JIA $[29,30]$ is an increased energy inside the damaged cells by enhanced ATP production and, externally, by stimulating vasodilation and increasing the rate at which nutrients are delivered, and waste products and cellular debris are removed from injured cells, which may reduce edema and other inflammatory reactions.

Obtaining a state of inactive disease induced by laser sublingually applied allows avoiding the aggressive immunosuppressive drugs (biological agents) to children in development and the high treatment costs.

Methotrexate and SLBI were well tolerated by JIA patients and yielded optimal outcomes. Mixed benefits from MTX and SLBI possibly indicate changes in the underlying biology of JIA.

\section{Conclusions}

At the end of the study, in SLBI group, there was an improvement of the ACR Pedi 30 by $86.11 \%$, compared to only $61.11 \%$ in Group II, respectively, and $60.6 \%$ in Group III $(P=0.001)$, with significant statistical differences. SLBI has reduced the pain, lowered the number of articulations with movement limitation, increased the quality of life, and made it possible to avoid the administration of biological agents.
We could conclude that SLBI as add-on Methotrexate therapy had a significant impact on the JIA ACR responses observed in our study.

SLBI is easy, noninvasive, and with high absorption on intensely vascularized buccal mucosa, triggering rapid systemic effects.

Our study could open the perspective of multiple treatment options in immune-mediated inflammatory disease using photobiomodulation, as an area of innovative and novel research with significant clinical implications.

\section{Disclosure}

The investigations were performed using a system from Weber Medical GmbH; however, there was no financial support from the company.

\section{Conflict of Interests}

The authors declare that there is no conflict of interests regarding the publication of this paper.

\section{Acknowledgments}

The authors would like to thank Ms. Ingrid Gaischek, M.S. (Medical University of Graz), for paper and figure preparation. The study was supported by the Austrian Federal Ministries of Science, Research and Economy and of Health (project title "Evidence-based high-tech acupuncture and integrative laser medicine for prevention and early intervention of chronic diseases") and the German Academy of Acupuncture (DAA).

\section{References}

[1] M. L. Stoll and R. Q. Cron, "Treatment of juvenile idiopathic arthritis: a revolution in care," Pediatric Rheumatology, vol. 12, article 13, 2014.

[2] R. Q. Cron, P. Weiser, and T. Beukelman, "Juvenile idiopathic arthritis," in Clinical Immunology: Principles and Practice, R. R. Rich, T. A. Fleisher, W. T. Shearer, H. Schroeder, A. J. Frew, and C. M. Weyand, Eds., pp. 637-647, Elsevier, London, UK, 4th edition, 2013.

[3] H. J. Chang, A. E. Burke, and R. M. Glass, "Juvenile idiopathic arthritis," Journal of the American Medical Association, vol. 303, no. 13, article 1328, 2010.

[4] C. Ailioaie and L. M. Ailioaie, "Juvenile idiopathic arthritis," in Management of Chronic Rheumatic Pain, pp. 129-146, PIM Publishing House, Iasi, Romania, 2008.

[5] M. Zak and F. K. Pedersen, "Juvenile chronic arthritis into adulthood: a long-term follow-up study," Rheumatology, vol. 39, no. 2, pp. 198-204, 2000.

[6] A. M. Prieur and G. Chèdeville, "Prognostic factors in juvenile idiopathic arthritis," Current Rheumatology Reports, vol. 3, no. 5, pp. 371-378, 2001.

[7] L. M. Ailioaie, C. Ailioaie, R. M. Chirieac, and A. Chirieac, "Effects of physical and vibroacoustic therapy in chronic pain in juvenile arthritis," Revista Romanade Reumatologie, vol. 20, no. 3, pp. 198-202, 2011. 
[8] A. Goodman, "Early aggressive therapy succeeds in children with arthritis," Medscape Medical News, 2013, http://www. medscape.com/viewarticle/814216.

[9] T. Beukelman, F. Xie, L. Chen et al., "Rates of hospitalized bacterial infection associated with juvenile idiopathic arthritis and its treatment," Arthritis \& Rheumatism, vol. 64, no. 8, pp. 2773-2780, 2012.

[10] M. Weber, "The intravenous laser blood irradiation. Introduction of a new therapy," in Lasers in Medicine, Science and Praxis, Z. Simunovic, Ed., pp. 664-706, Zrinski Printery Publishing House, Cakovec, Croatia, 2009.

[11] D. A. Chiran, G. Litscher, M. Weber, L. M. Ailioaie, C. Ailioaie, and D. Litscher, "Intravenous laser blood irradiation increases efficacy of etanercept in selected subtypes of juvenile idiopathic arthritis: an innovative clinical research approach," EvidenceBased Complementary and Alternative Medicine, vol. 2013, Article ID 168134, 9 pages, 2013.

[12] D. A. Chiran, M. Weber, L. M. Ailioaie et al., "Intravenous laser blood irradiation and tocilizumab in a patient with juvenile arthritis," Case Reports in Medicine, vol. 2014, Article ID 923496, 6 pages, 2014.

[13] L. N. Moorthy, M. Peterson, A. L. Hassett, and T. Lehman, "Burden of childhood-onset arthritis," Pediatric Rheumatology, vol. 8, article 20, 2010.

[14] M. H. Otten, J. Anink, F. H. Prince et al., “Trends in prescription of biological agents and outcomes of juvenile idiopathic arthritis: results of the Dutch national Arthritis and Biologics in Children Register," Annals of the Rheumatic Diseases, 2014.

[15] R. E. Petty, T. R. Southwood, P. Manners et al., "International League of Associations for Rheumatology classification of juvenile idiopathic arthritis: second revision, Edmonton, 2001," Journal of Rheumatology, vol. 31, no. 2, pp. 390-392, 2004.

[16] E. H. Giannini, N. Ruperto, A. Ravelli, D. J. Lovell, D. T. Felson, and A. Martini, "Preliminary definition of improvement in juvenile arthritis," Arthritis \& Rheumatism, vol. 40, no. 7, pp. 1202-1209, 1997.

[17] H. I. Brunner, D. J. Lovell, B. K. Finck, and E. H. Giannini, "Preliminary definition of disease flare in juvenile rheumatoid arthritis," Journal of Rheumatology, vol. 29, no. 5, pp. 1058-1064, 2002.

[18] G. Singh, B. H. Athreya, J. F. Fries, and D. P. Goldsmith, "Measurement of health status in children with juvenile rheumatoid arthritis," Arthritis \& Rheumatism, vol. 37, no. 12, pp. 1761-1769, 1994.

[19] N. Ruperto, A. Ravelli, A. Pistorio et al., "Cross-cultural adaptation and psychometric evaluation of the Childhood Health Assessment Questionnaire (CHAQ) and the Child Health Questionnaire (CHQ) in 32 countries. Review of the general methodology," Clinical and Experimental Rheumatology, vol. 19, supplement 23, pp. S1-S9, 2001.

[20] D. S. Goodsell, “The molecular perspective: methotrexate," The Oncologist, vol. 4, no. 4, pp. 340-341, 1999.

[21] P. T. R. Rajagopalan, Z. Zhang, L. McCourt, M. Dwyer, S. J. Benkovic, and G. G. Hammes, "Interaction of dihydrofolate reductase with methotrexate: ensemble and single-molecule kinetics," Proceedings of the National Academy of Sciences of the United States of America, vol. 99, no. 21, pp. 13481-13486, 2002.

[22] J. A. M. Wessels, T. W. J. Huizinga, and H.-J. Guchelaar, "Recent insights in the pharmacological actions of methotrexate in the treatment of rheumatoid arthritis," Rheumatology, vol. 47, no. 3, pp. 249-255, 2008.
[23] L. M. Ailioaie, Contributions to the therapy of chronic pain in juvenile idiopathic arthritis by physical means and alternative medicine [Ph.D. thesis], Gr. T. Popa" University of Medicine and Pharmacy, Iasi, Romania, 2012.

[24] P. Avci, A. Gupta, M. Sadasivam et al., "Low-Level Laser (Light) Therapy (LLLT) in skin: stimulating, healing, restoring," Seminars in Cutaneous Medicine and Surgery, vol. 32, pp. 41-52, 2013.

[25] T. I. Karu and S. F. Kolyakov, "Exact action spectra for cellular responses relevant to phototherapy," Photomedicine and Laser Surgery, vol. 23, no. 4, pp. 355-361, 2005.

[26] M. Greco, G. Guida, E. Perlino, E. Marra, and E. Quagliariello, "Increase in RNA and protein synthesis by mitochondria irradiated with helium-neon laser," Biochemical and Biophysical Research Communications, vol. 163, no. 3, pp. 1428-1434, 1989.

[27] T. I. Karu, L. V. Pyatibrat, and G. S. Kalendo, "Photobiological modulation of cell attachment via cytochrome c oxidase," Photochemical and Photobiological Sciences, vol. 3, no. 2, pp. 211216, 2004.

[28] G. Haskó, J. Linden, B. Cronstein, and P. Pacher, "Adenosine receptors: therapeutic aspects for inflammatory and immune diseases," Nature Reviews Drug Discovery, vol. 7, no. 9, pp. 759$770,2008$.

[29] C. Ailioaie and L. M. Ailioaie, "Beneficial effects of laser therapy in the early stages of rheumatoid arthritis onset," Laser Therapy, vol. 11, no. 2, pp. 79-87, 1999.

[30] C. Ailioaie and L. M. Ailioaie, "Laser photobiostimulation and safety in pediatric diseases," in Lasers in Medicine, Science and Praxis, Z. Simunovic, Ed., chapter 32, pp. 467-504, Printery Publishing House, Cakovec, Croatia, 2009. 

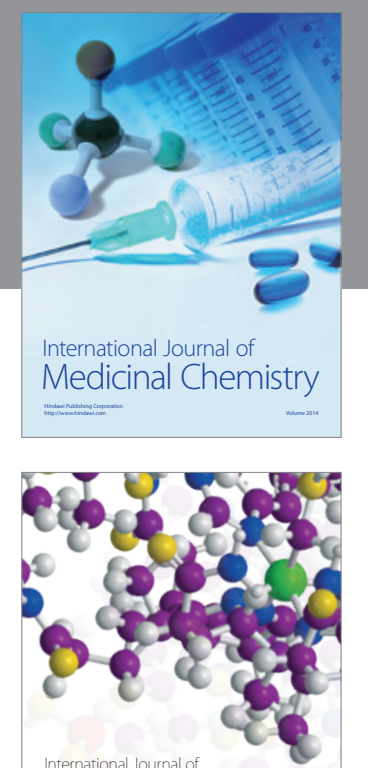

\section{Carbohydrate} Chemistry

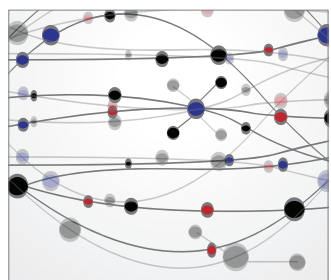

The Scientific World Journal
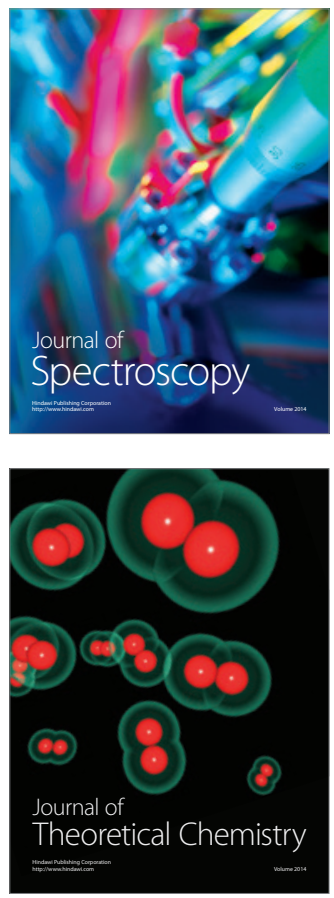
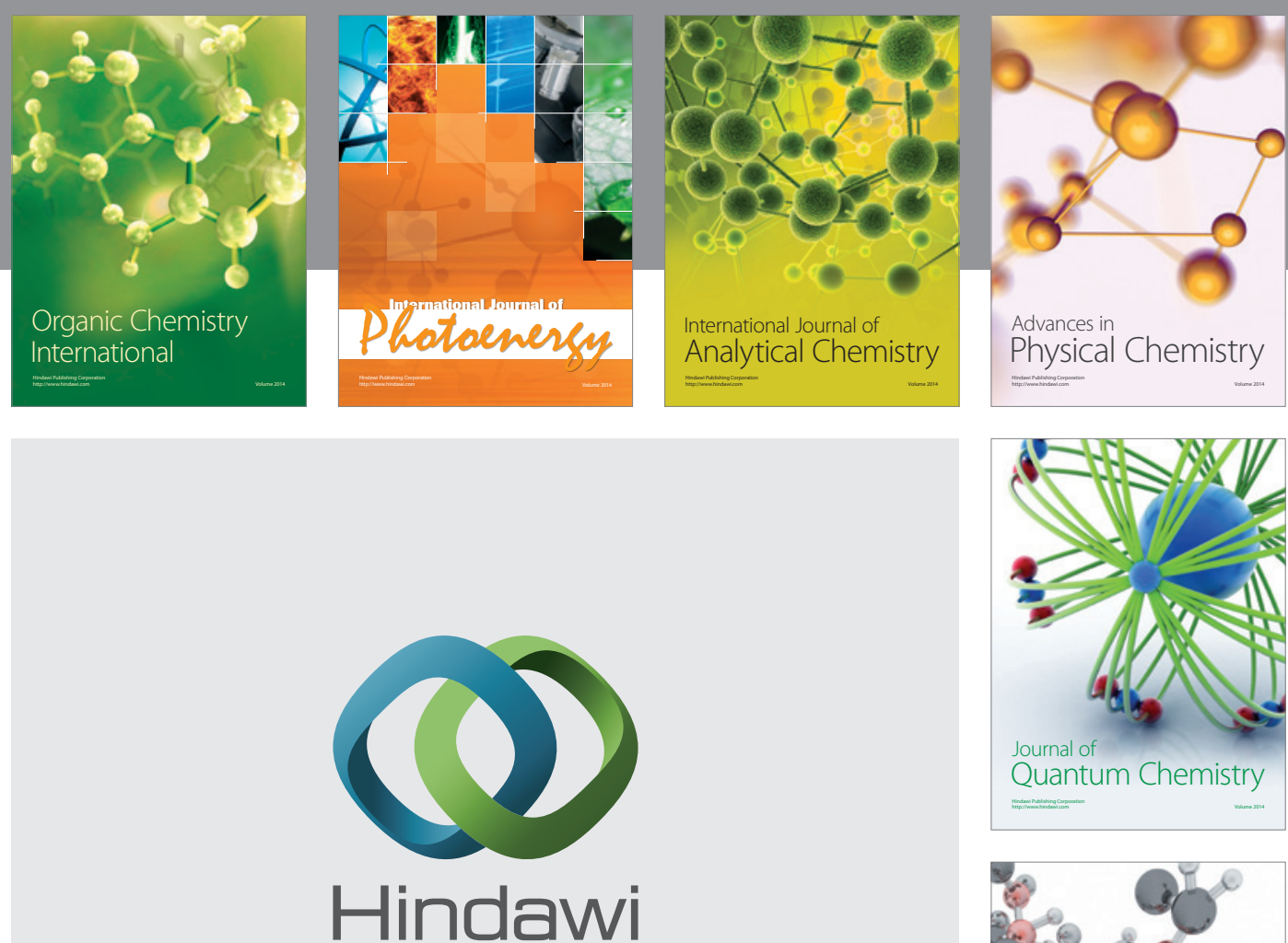

Submit your manuscripts at

http://www.hindawi.com

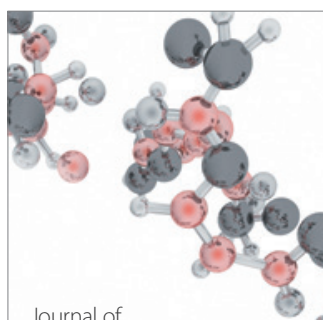

Analytical Methods

in Chemistry

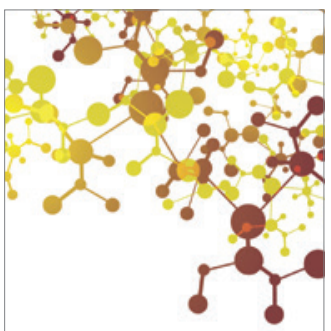

Journal of

Applied Chemistry

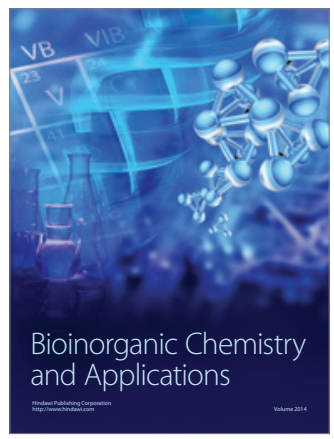

Inorganic Chemistry
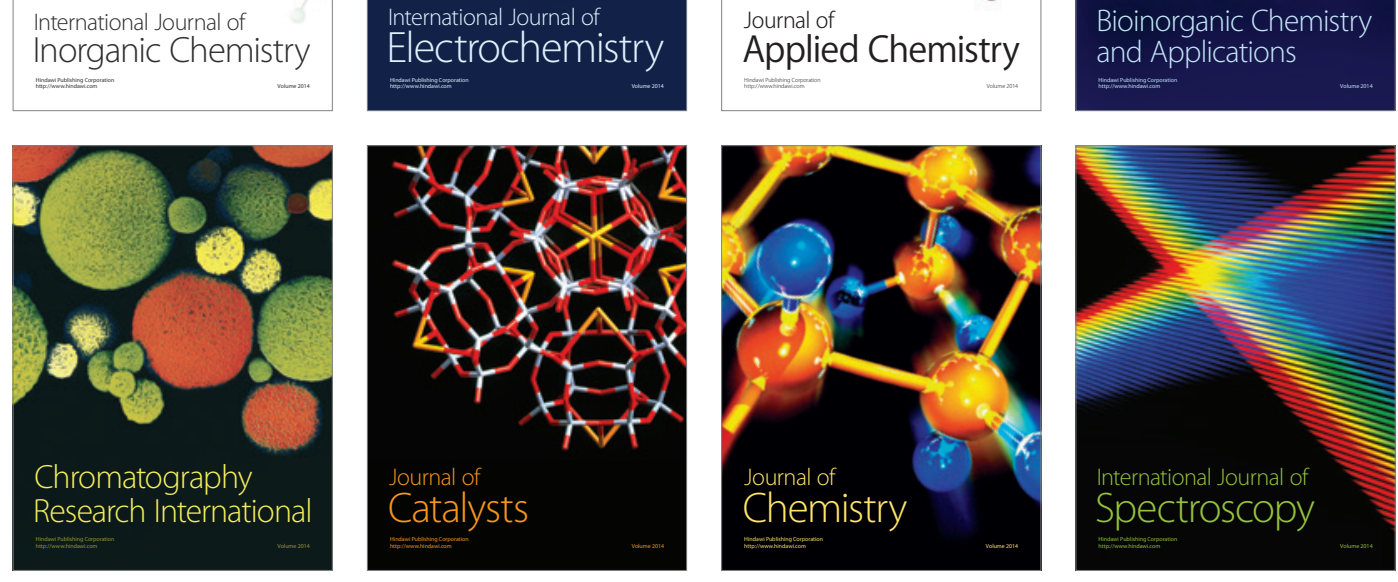